\title{
Safety efficacy and tolerability of the calcitonin gene-related peptide monoclonal antibodies for migraine prevention in a real world setting
}

Jasem Yousef Al-Hashel ( $\boldsymbol{D}$ dralhashel@hotmail.com )

Kuwait University Faculty of Medicine https://orcid.org/0000-0001-6371-7381

Raed Alroughani

Amiri Hospital

Hasan Kh Ashkanani

Farwaniya Hospital

Ohood AlMutairi

Farwaniya Hospital

Amr Akl

Farwaniya Hospital

\section{Sawsan Alwazzan}

Kuwait University Faculty of Medicine

\section{Samar Farouk Ahmed}

Ibn Sina Hospital;Kuwait. Minia University; Faculty of medicine, Egypt.

\section{Research article}

Keywords: Migraine, calcitonin gene-related peptide monoclonal antibodies, Kuwait

Posted Date: February 26th, 2021

DOl: https://doi.org/10.21203/rs.3.rs-266202/v1

License: (9) This work is licensed under a Creative Commons Attribution 4.0 International License. Read Full License 


\section{Abstract}

\section{Background:}

Calcitonin gene-related peptide (CGRP) monoclonal antibodies were approved as preventive treatment for migraine. We aimed to assess CGRP monoclonal antibodies efficacy and safety.

\section{Methods:}

Patients were diagnosed based on International Classification of Headache Disorders disorders-third edition, (ICHD-III). Eligible patients received CGRP monoclonal antibodies (mAbs) and had a follow up visits for at least three months. Primary end point was a $50 \%$ or greater reduction in mean migraine days per month at the last visit. Secondary end points were change from baseline to last visit in the mean number of migraine days per month, change in the number of days of use of analgesics, change in the severity of headache pain, and change in scores on the Quality of life (QOL) questionnaire. Safety and tolerability were reported.

\section{Results:}

This prospective study included 63 migraine patients, with a mean age of 44.59 years. Females represented $77.8 \%$ of the cohort. A $50 \%$ or greater reduction in the mean number of migraine days per month was achieved in $68.3 \%$ of patients. At the last visit, patients had a significant reduction in migraine days, number of days of analgesic-usage and the severity of headache $(5.13 \pm 4.11$ versus $14.04 \pm 7.77 ; p$ $<0.01),(6.43 \pm 6.34$ versus $15.85 \pm 7.31 ; p<.0001),(5.40 \pm 2.48$ versus $9.67 \pm 0.72 ; p<0.001)$ respectively. CGRP mAb treatment significantly improved quality of life $(112.20 \pm 12.20$ versus $92.13 \pm 15.84 ; \mathrm{P}<$ 0.001). $14.4 \%$ reported adverse events.

\section{Conclusion:}

Prophylactic CGRP monoclonal antibodies treatment for migraine were efficacious and reduced the number of monthly migraine days and the use of days of analgesics.

\section{Introduction}

Migraine, characterized by recurrent attacks of headache, and it is a common disabling neurological disorder usually associated with nausea, vomiting, photophobia or phonophobia. [1] Headache disorders, mainly including migraine, ranked second in the leading causes of disability worldwide according to the latest Global Burden of Disease Study. Migraine headache is prevalent in Kuwait. Our previous study showed that prevalence of episodic migraine was $23.11 \%$, chronic migraine $5.4 \%$, and medication overuse headache $2.4 \%[4]$.

The approach in the treatment of migraines include treating the acute attacks and preventing future attacks with preventive therapy. [3] Migraine in Kuwait has a significant impact on daily living activities, 
schooling/ employment and social lives of patients[5], thus showing the importance of preventive therapy. The use of OnabotulinumtoxinA, and Topiramate have been the focus of studying preventive therapy in migraine headaches with good quality evidence [6-7]. The management of patients with migraine might be unsatisfactory due to lack of efficacy and intolerable adverse events of available conventional preventive therapies. Thus, novel effective drugs with good tolerability, few side effects and high retention rates are needed for migraineurs [8].

During migraine attack, Calcitonin gene-related peptide (CGRP) has been shown to be at high levels in jugular venous blood. [9] CGRP was also elevated in the blood of migraine patients not experiencing an attack giving the future possibility of using CGRP as a biomarker in the diagnosis of migraines. $[10,11]$ This observation has led to the focus on the CGRP pathway in the treatment of migraines. CGRP, which is a proinflammatory vasodilating neuropeptide has been found to play an important role in the pathophysiology of migraine via nociceptive mechanisms in the trigeminovascular system. Its role in pathophysiology of migraine directed the focus of preventive therapy to it. [12]

Monoclonal antibodies against the CGRP pathway (anti-CGRP) have been developed for the prevention of episodic migraine and chronic migraine, either by binding to CGRP ligand (fremanezumab, galcanezumab, eptinezumab) or receptor (erenumab). [13-14]

Galcanezumab is humanized monoclonal antibody (mAb) that potently and selectively bind to CGRP, while erenumab is the only mAb that targets CGRP receptor instead of CGRP ligand. [15] There are randomized clinical trials (RCTs) that evaluate these drugs [16-21]

The US Food and Drug Administration (FDA) approved Erenumab, an anti-CGRP receptor monoclonal antibody (mAb), for the prevention of migraine on May 17, 2018. and on September 26, 2018, the FDA approved galcanezumab, anti-CGRP ligand monoclonal antibodies for the prevention of migraine[22, 23]. Erenumab is a monthly subcutaneous injections of either $70 \mathrm{mg}$ or $140 \mathrm{mg}$ [13]. Galcanezumab is a humanized IgG4 mAb that binds to both $\alpha$ and $\beta$ forms of the human CGRP ligand. It's a monthly subcutaneous injection of $120 \mathrm{mg}$ with a first loading dose of $240 \mathrm{mg}[14]$. Both are registered and available in Kuwait.

Here, we aim to assess CGRP mAb efficacy and safety in real setting in Kuwait.

\section{Methods}

This prospective, questionnaire-based study was conducted in a specialized headache clinic in a tertiary hospital in Kuwait. The study population included both male and female patients aged 18-65 years who are diagnosed with chronic migraine (CM) with or without medication over use headache $(\mathrm{MOH})$. Diagnoses were confirmed by headache specialist according to International Classification of Headache Disorders III (ICHD-III) [1]. The study excluded patients with psychiatric disorders or chronic medical problems. 
Patients with a diagnosis of another headache disorder, previous use of CGRP mAb for treatment of any headache, and pregnant or breast-feeding patients were excluded from the study.

The study included patients who received CGRP mAb, being either erenumab or galcanezumabthat, that are available in Kuwait. Eligible patients received CGRP mAb every 4 weeks subcutaneously and had follow up visits for at least three months. The primary end point was a $50 \%$ or greater reduction in mean migraine days per month in their last visit. Secondary end points were the change from baseline to last visit in the mean number of migraine days per month, change in the number of days of use of analgesics, change in the severity of headache pain assessed by scores of the visual analogue scale (VAS), and change in scores on the Quality of life questionnaire. Safety and tolerability were reported in this study as well.

This study was carried out in accordance with the ethical guidelines of Kuwait Ministry of Health. The protocol was approved by the ethical committee of Ibn Sina hospital.

All subjects gave a written informed consent in accordance with the Declaration of Helsinki [24].

The study was performed in observation of the latest version of the declaration of Helsinki, and all data was anonymous and protected in accordance with the ethical guidelines of the Council for International Organizations of Medical Sciences.

Statistical analyses were performed with IBM SPSS Statistics 20.0 software for Mac (SPSS Inc., Chicago, IL, USA). All continuous variables were expressed as means, whereas categorical ones were expressed as proportions and percentages. Paired sample t test was used to compare between variables. Chi-square test was adopted for categorical variables. A significant difference was set to be at $p<0.05$.

\section{Results}

A total of 63 patients were included in this study. Table 1 displays Demographic and clinical characters of our cohort. The mean age of the participants and disease onset were 44.5 and 22.8 years, respectively. The study was representative of both males and females, with the latter accounting for $77.8 \%$ of the sample size. The majority of the patients had a positive family history of migraine (63.5\%). Most of our cohort (63.5\%) started calcitonin gene-related peptide mAb due to of lack of efficacy, or due to adverse events of previous prophylactic medications in (20.6\%). The patients were treated with calcitonin generelated peptide $\mathrm{mAb}$ for a mean of 7.5 months and almost $85 \%$ of them have taken at least one previous prophylactic medication. 
Table 1

Demographic and clinical characters of migraine patients on CGRP mAbs $(\mathrm{N}=63)$.

\begin{tabular}{|c|c|}
\hline Variables & Mean \pm SD/Number (\%) \\
\hline Mean Age in years & $44.59 \pm 11.71$ \\
\hline Range & $18-66$ \\
\hline Mean Age at onset of migraine & $22.87 \pm 8.21$ \\
\hline Range & $6-52$ \\
\hline Gender & $14(22.2)$ \\
\hline • Male & $49(77.8)$ \\
\hline \multicolumn{2}{|l|}{ - Female } \\
\hline Mean Disease duration in years & $21.87 \pm 12.51$ \\
\hline Range & $2-50$ \\
\hline Family history of migraine & $40(63.5)$ \\
\hline Diagnosis & $27(42.9)$ \\
\hline - Episodic migraine & $5(7.9)$ \\
\hline • Chronic migraine & $31(49.2)$ \\
\hline \multicolumn{2}{|l|}{ - Medication over use headache } \\
\hline Mean Treatment duration in months & $7.57 \pm 1.42$ \\
\hline Range & $3-12$ \\
\hline Comorbidity & $30(47.6)$ \\
\hline - No comorbidity & $11(17.5)$ \\
\hline •DM/HTN & $7(11.1)$ \\
\hline - Psychiatric disorders & $11(17.5)$ \\
\hline - Obesity & $4(6.3)$ \\
\hline \multicolumn{2}{|l|}{ - Others } \\
\hline Previous use of prophylactic medications & $54(85.7)$ \\
\hline - Yes & $9(14.3)$ \\
\hline • no & \\
\hline
\end{tabular}

CGRP: Calcitonin gene-related peptide; DM: diabetes mellitus; HTN: hypertension; mAbs: Monoclonal antibodies; N: number; SD: slandered deviation. 


\begin{tabular}{|ll|}
\hline Variables & Mean \pm SD/Number (\%) \\
\hline Number of previous prophylactic medication & $9(14.3)$ \\
- No prophylactic medication & $18(28.6)$ \\
- One prophylactic medication & $10(15.90$ \\
- Two prophylactic medication & $8(12.7)$ \\
- Three prophylactic medication & $18(28.6)$ \\
- Four prophylactic medication & \\
\hline Cause of starting on CGRP mAbs & $13(20.6)$ \\
- Adverse events of previous prophylactic medication & $40(63.5)$ \\
- Lack of efficacy of prophylactic medication & $2(3.2)$ \\
- Adherence & $8(12.7)$ \\
• Patient preference & \\
\hline $\begin{array}{l}\text { CGRP: Calcitonin generelated peptide; DM: diabetes mellitus; HTN: hypertension; mAbs: Monoclonal } \\
\text { antibodies; N: number; SD: slandered deviation. }\end{array}$ \\
\hline
\end{tabular}

Table 2 displays primary and second end points. The majority of the participants (68.3\%) had at least $50 \%$ reduction in the number of migraine days per month. Table 2 showed significant reduction in the mean number of days with migraine per month $(14.04+-7.77$ to $5.13+-4.11$ days; $p=0.01)$. In addition, the mean number of days with analgesic usage significantly decreased (15.85 +- 7.31 to $6.43+-6.34$ days; $p=0.001)$. Moreover, the severity of headache, as assessed by pain scale, has significantly improved $(9.67+-0.72$ to $5.4+-2.48 ; p=0.001)$. A significant improvement in quality of life was also noted $(92.13+-15.84$ vs $112.20+-12.2, p=0.001)$. At last visit all chronic migraine patients and $80.6 \%$ patients with $\mathrm{MOH}$ converted to episodic migraine (Table 2). The frequency of episodic migraine, chronic migraine, and medication overuse headache before and after treatment was ( $42.9 \%$ vs $90.5 \%)$, ( $7.9 \%$ vs $0 \%)$, and ( $49.2 \%$ vs $9.5 \%)$, respectively. This difference in frequency was statistically significant for all groups $(p<0.001)$. 
Table 2

Efficacy and impact of CGRP mAbs on migraine headache patients $(N=63)$.

\begin{tabular}{|llll|}
\hline Variables & $\begin{array}{l}\text { Migraine state before } \\
\text { CGRP mAbs } \\
\text { treatment } \\
\text { Mean } \pm \text { SD/Number } \\
(\%)\end{array}$ & $\begin{array}{l}\text { Migraine state at last visit } \\
\text { after CGRP mAbs } \\
\text { treatment } \\
\text { Mean } \pm \text { SD/Number (\%) }\end{array}$ & P \\
\hline $\begin{array}{l}\text { Primary end point: Reduction 50\% in } \\
\text { the number of migraine days per } \\
\text { month. }\end{array}$ & $43(68.3)$ & & \\
\hline $\begin{array}{l}\text { Secondary end points } \\
\text { Migraine days/month }\end{array}$ & $14.04 \pm 7.77$ & $5.13 \pm 4.11$ & $0.01^{*}$ \\
Headache days/months & $5.22 \pm 7.58$ & 3.226 .40 & $0.001^{*}$ \\
Analgesic days/month & $15.85 \pm 7.31$ & $6.43 \pm 6.34$ & $0.001^{*}$ \\
Severity of headache & $9.67 \pm 0.72$ & $5.40 \pm 2.48$ & $0.001^{*}$ \\
Quality of life & $92.13 \pm 15.84$ & $112.20 \pm 12.20$ & $0.001^{*}$ \\
Diagnosis & $27(42.9)$ & $57(90.5)$ & $0.001^{*}$ \\
• Episodic migraine & $5(7.9)$ & 0 & \\
• Chronic migraine & $31(49.2)$ & $6(9.5)$ & \\
\hline - Medication over use headache & & & \\
\hline $\begin{array}{l}\text { *: statistically significant; CGRP: Calcitonin gene-related peptide; mAbs: Monoclonal antibodies; N: } \\
\text { number; SD: slandered deviation. }\end{array}$ & & & \\
\hline
\end{tabular}

The majority of the participants did not experience any side effects related to calcitonin gene-related peptide antibodies treatment (84.1\%) (Table 3). The most common side effects reported were pain at the injection site (6.4\%) and constipation (6.4\%). Other side effects were palpitations (1.6\%) and psychological symptoms (1.7\%). In addition, $71.4 \%$ of the patients were satisfied with the medication and $66.7 \%$ were compliant to the treatment. However, discontinuation of treatment was seen in $9.6 \%$ of the participants. Although side effects occurred in $15.9 \%$ of the study participants, they were not reported as a cause of discontinuation. On the other hand, shortage of medication and lack of efficacy were the most common reasons for stopping the treatment $(6.4 \%$ and $3.2 \%$, respectively). 
Table 3

Outcome of CGRP mAbs on migraine headache patients $(\mathrm{N}=63)$.

\begin{tabular}{|ll|}
\hline Variables & Mean/SD, Number (\%) \\
\hline Satisfaction & $45(71.4)$ \\
\hline Compliance & $42(66.7)$ \\
\hline Adverse events & $53(84.1)$ \\
- No & $4(6.4)$ \\
- Injection site pain & $4(6.4)$ \\
- Constipation & $1(1.6)$ \\
- Palpitation & $1(1.7)$ \\
- Psychological symptoms & \\
\hline Discontinuation & $6(9.6)$ \\
- Adverse events & 0 \\
• Lack of efficacy & $2(3.2)$ \\
• Shortage of medication & $4(6.4)$ \\
\hline $\begin{array}{l}\text { CGRP: Calcitonin gene-related peptide; mAbs: Monoclonal antibodies; N: number; SD: slandered } \\
\text { deviation. }\end{array}$ \\
\hline
\end{tabular}

\section{Discussion}

This study included 63 migraine patients who used CGRP mAbs for mean of 7 months. It is worth that, the majority of our participants (85\%), had already unsuccessfully tried at least one prophylactic medication to treat their migraine. Our results found that $68 \%$ of participants had $50 \%$ or greater reduction in the mean number of migraine days per month. At last visit, patients had a significant reduction in mean migraine days, mean number of days of analgesic-usage and the severity of headache. This confirms that CGRP mAbs treatment is effective in migraine patients who had a history of unsuccessful or inadequate treatment. In previous studies, CGRP mAb also showed efficacy in treating migraine patients who failed multiple first line migraine preventive therapies. [18, 25]. Our results are similar to previous studies showing the effects of CGRP mAb in migraine prevention. [16-21, 25, 26]. The number of days with analgesic usage decreased along with the severity of headaches reported by the patients in this study. CGRP mAbs relived migraine pain and this is explained by reduction or normalization of CGRP concentrations in brain tissue and blood after treatment CGRP receptor agonists or also Botulinum toxin.[27,28].

In our study, secondary end-points showed that CGRP mAb was able to reduce monthly migraine days by a mean of 9 days in more than $50 \%$ of the patients. In addition, monthly analgesic days were reduced by 
a mean of 10 days. The severity of headache and quality of life were improved by a mean of 5 and 20 points on pain severity scale and QOL assessment questionnaire, respectively, as reported by the patients. All patients who were diagnosed with chronic migraine met the ICHD-III criteria for episodic migraine after CGRP mAbs treatment, reflecting a significant reduction in the frequency of migraine headache.

Improvement in the QOL, confirm that the reduction of migraine days with using CGRP mAbs decreased the burden of the disease on the patients. This was similar to the previous studies [29].

Our results confirm the efficacy of CGRP mAbs treatment for migraine headache. The number of chronic migraine patients in our study (5 patients) was relatively small, hence, a solid conclusion cannot be drawn from such small sample size. We also found that the mean reduction in monthly migraine days (9 days) was larger than what was found in the previous studies $[16,17.20,21]$.

Our study also included patients with medication overuse headache. CGRP mAbs treatment decreased the percentage of those patients from 49.2-9.5\%. Most of these patients met the criteria for episodic migraine after treatment with CGRP mAbs. Our result is in line with result of Tepper who reported that erenumab resulted in a significant reduction in acute migraine- specific medication treatment days [29].This considerable reduction in the number of patients with medication overuse headache indicates that CGRP mAbs treatment might be effective in treating comorbid medication overuse headache associated with migraine prophylactic treatment.

The low discontinuation rate reported here (9.6\%) shows that CGRP mAbs has good tolerability adding to the advantages of using it as a preventive therapy. Only $15.9 \%$ of the participants reported side effects after using CGRP mAbs, such as pain at the injection site (6.4\%), constipation (6.4\%), palpitations (1.6\%) and psychological symptoms (1.7\%); with no life-threatening adverse effects reported. None of the side effects reported here were a reason for discontinuation of treatment and they are similar to the ones reported in multiple similar studies [18, 30,31]. CGRP mAbs had a favorable safety profile, and there were no specific adverse events as seen in previous results [16-21, 32].

\section{Limitation Of The Study}

The study was limited by the small number of the sample, especially chronic migraine patients. This drawback decreases the power of our study. Also the primary and secondary end-points were assessed subjectively based on the patients' recall, in which recall bias can occur.

\section{Conclusion}

Prophylactic CGRP mAbs treatment for migraine reduce the numbers of monthly migraine days and acute migraine-specific medication days, as well as improve improved quality of life. They may convert chronic migraine to episodic migraine and reduce frequency of $\mathrm{MOH}$. They were well tolerated and incidence of treatment withdrawal rate was low. 


\section{Abbreviations}

Calcitonin gene-related peptide: CGRP

Chronic migraine: $\mathrm{CM}$

Diagnostic and Statistical Manual of Mental Disorders: DSM-5

Food and Drug Administration: FDA

International Classification of Headache Disorders III: ICHD-III

Medication over use headache: $\mathrm{MOH}$

Monoclonal antibodies: mAbs

Quality of Life: QOL

\section{Declarations}

This research was carried out according to ethical guidelines of Kuwait Ministry of Health.

\section{Ethic Approval and Patient Consent}

The Institutional Review Board Committee of Ministry of Health of the State of Kuwait approved this study. All subjects gave written informed consent in accordance with the Declaration of Helsinki.

\section{STRENGTH OF THE STUDY}

Our study is the first one that estimated the safety and efficacy of CGRP monoclonal antibodies in Middle East. It also included patients with medication overuse headache and showed to provide some benefit.

\section{Ethics approval and consent to participate}

Not applicable

\section{Consent for publication}

Not applicable

\section{Availability of data}

Data are available at administrative section, neurology department, Ibn Sina hospital, Kuwait.

\section{Competing interests}

No competing interests. 


\section{Funding}

The study is not funded.

\section{Authors' contributions}

JA-H designed the study and reviewed the manuscript. RA drafted and reviewed the manuscript. HKA, AK, OA, SA performed data collection and drafted the manuscript. SFA performed statistical analysis, drafted, criticized and reviewed the manuscript. All authors read and approved the final manuscript.

\section{Acknowledgements}

We would like to thank the study participants and the administrative department in Ibn Sina Hospital for supporting this study. Our appreciation to nurse Samia Omar and nurse Enayaat Khairy at headache clinic in Ibn Sina Hospital for actively supporting this study.

\section{Conflicts of Interest}

None declared.

\section{References}

1. Arnold M. Headache classification committee of the international headache society (ins) the international classification of headache disorders. Cephalalgia 2018;38(1):1-211.

2. Stovner LJ, Nichols E, Steiner TJ, Abd-Allah F, Abdelalim A, Al-Raddadi RM, Ansha MG, Barac A, Bensenor IM, Doan LP, Edessa D. Global, regional, and national burden of migraine and tension-type headache, 1990-2016: a systematic analysis for the Global Burden of Disease Study 2016. The Lancet Neurology 2018 Nov 1;17(11):954-76.

3. Goadsby PJ, Sprenger T. Current practice and future directions in the prevention and acute management of migraine. Lancet Neurology 2010 Mar;9(3):285-98. doi: 10.1016/S14744422(10)70005-3. PMID: 20170842.

4. Al-Hashel JY, Ahmed SF, Alroughani R. Prevalence of primary headache disorders in Kuwait. Neuroepidemiology 2017;48(3-4):138-46.

5. Al-Hashel JY, Ahmed SF, Alroughani R. Burden of migraine in a Kuwaiti population: a door-to-door survey. The journal of headache and pain 2017; 18(1):1-6.

6. Dodick DW, Turkel CC, DeGryse RE, et al; PREEMPT Chronic Migraine Study Group. OnabotulinumtoxinA for treatment of chronic migraine: pooled results from the double-blind, randomized, placebo-controlled phases of the PREEMPT clinical program. Headache 2010 Jun;50(6):921-36. doi: 10.1111/j.1526-4610.2010.01678.x. Epub 2010 May 7. PMID: 20487038.

7. Naegel S, Obermann M. Topiramate in the prevention and treatment of migraine: efficacy, safety and patient preference. Neuropsychiatr Dis Treat 2010 Feb 3;6:17-28. doi: 10.2147/ndt.s6459. PMID: 
$20169042 ;$ PMCID: PMC2951059.

8. Deng H, Li GG, Nie H, et al. Efficacy and safety of calcitonin-gene-related peptide binding monoclonal antibodies for the preventive treatment of episodic migraine-an updated systematic review and meta-analysis. BMC neurology 2020 Dec;20(1):1-2.

9. Goadsby PJ, Edvinsson L, Ekman R. Vasoactive peptide release in the extracerebral circulation of humans during migraine headache. Ann Neurolology 1990 Aug;28(2):183-7. doi:

10.1002/ana.410280213. PMID: 1699472.

10. Ashina M, Bendtsen $L$, Jensen R, et al. Evidence for increased plasma levels of calcitonin generelated peptide in migraine outside of attacks. Pain 2000 May;86(1-2):133-8. doi: 10.1016/s03043959(00)00232-3. PMID: 10779670.

11. Cernuda-Morollón E, Larrosa D, Ramón C, et al. Interictal increase of CGRP levels in peripheral blood as a biomarker for chronic migraine. Neurology 2013 Oct 1;81(14):1191-6. doi:

10.1212/WNL.0b013e3182a6cb72. Epub 2013 Aug 23. PMID: 23975872.

12. Edvinsson L. The Trigeminovascular pathway: role of CGRP and CGRP receptors in migraine. Headache2017;57(Suppl 2):47-55. doi: 10.1111/head.13081.

13. Committee for Medicinal Products for Human Use (CHMP). Aimovig, INN-erenu- mab. European Medicines Agency. Assessment report [Internet] 2018 [accessed 12/25/2019]. Available at: ema.europa.eu/contact

14. Committee for Medicinal Products for Human Use (CHMP). Emgality, INN-galcane- zumab. European Medicines Agency. Assessment report [Internet]. 2018 [accessed 12/25/2019]. Available at: www.ema.europa.eu/contact

15. Deng H, Li GG, Nie H, et al. Efficacy and safety of calcitonin-gene-related peptide binding monoclonal antibodies for the preventive treatment of episodic migraine-an updated systematic review and meta-analysis. BMC neurology 2020 Dec;20(1):1-2.

16. Tepper S, Ashina M, Reuter U, et al. Safety and efficacy of erenumab for preventive treatment of chronic migraine: a randomised, double-blind, placebo-controlled phase 2 trial. Lancet Neurol. 2017 Jun 1;16(6):425-34. DOI: 10.1016/S1474-4422(17)30083-2.

17. Silberstein SD, Dodick DW, Bigal ME, et al. Fremanezumab for the Preventive Treatment of Chronic Migraine. N Engl J Med 2017 Nov 30;377(22):2113-22. DOI: 10.1056/NEJMoa1709038

18. Reuter U, Goadsby PJ, Lanteri-Minet M, et al. Efficacy and tolerability of erenumab in patients with episodic migraine in whom two-to-four previous preventive treatments were unsuccessful: a randomised, double-blind, placebo-controlled, phase 3b study. Lancet 2018 Nov 24;392(10161):2280-7. DOI: 10.1016/S0140-6736(18)32534-0

19. Dodick DW, Silberstein SD, Bigal ME, et al. Effect of Fremanezumab compared with placebo for prevention of episodic migraine a randomized clinical trial. JAMA 2018 May 15;319(19):1999-2008. DOI: $10.1001 /$ jama.2018.4853

20. Dodick DW, Lipton RB, Silberstein S, et al. Eptinezumab for prevention of chronic migraine: A randomized phase 2b clinical trial. Cephalalgia 2019 Aug;39(9):1075-85. DOI: 
$10.1177 / 0333102419858355$

21. Detke HC, Goadsby PJ, Wang S, et al. Galcanezumab in chronic migraine: The randomized, doubleblind, placebo-con- trolled REGAIN study. Neurology 2018;91(24):E2211-21. DOI: 10.1212/ WNL.0000000000006640

22. Novartis and Amgen announce FDA approval of Aimovig(TM) (erenumab), a novel treatment developed specifically for migraine preventionhttps:// novartis.com/news/media-releases/novartisand-amgen-announce-fda- approval-aimovigtm-erenumab-novel-treatment-developed-specificallymigraine-prevention (accessed 20 September 2018)

23. Lilly's EmgalityTM (galcanezumab-gnIm) Receives U.S. FDA Approval for the Preventive Treatment of Migraine in Adultshttps://lilly.com/news- releases/news-release-details/lillys-emgalitytmgalcanezumab-gnlm-receives- us-fda-approval (accessed 31 October 2018)

24. World Medical Association (WMA): WMA Declaration of Helsinki - Ethical Principles for Medical Research Involving Human Subjects. 2001. http://www.wma. net/en/30pu-blications/10policies/b3/ (cited 2001).

25. Goadsby PJ, Reuter U, Hallström Y, et al. A controlled trial of erenumab for episodic migraine. New England Journal of Medicine 2017 Nov 30;377(22):2123-32.

26. Stauffer VL, Dodick DW, Zhang Q et al. Evaluation of Galcanezumab for the prevention of episodic migraine: the EVOLVE-1 randomized clinical trial. JAMA Neurol 2018; 75:1080-1088

27. Hou M, Xing H, Cai Y, et al. The effect and safety of monoclonal antibodies to calcitonin gene-related peptide and its receptor on migraine: a systematic review and meta-analysis. The journal of headache and pain 2017 Dec;18(1):42..

28. Silberstein SD. Review of botulinum toxin type A and its clinical applications in migraine headache. Expert Opin Pharmacother 2005; 2:1649-1654]

29. Tepper SJ, Diener HC, Ashina M, et al. Erenumab in chronic migraine with medication overuse: Subgroup analysis of a randomized trial. Neurology 2019 May 14;92(20):e2309-e2320. doi: 10.1212/WNL.0000000000007497. Epub 2019 Apr 17. PMID: 30996056; PMCID: PMC6598821.

30. Raffaelli B, Kalantzis R, Mecklenburg J, et al. Erenumab in chronic migraine patients who previously failed five first-line Oral prophylactics and OnabotulinumtoxinA: a dual-center retrospective observational study. Frontiers in neurology 2020;11.

31. Kanaan S, Hettie G, Loder E, et al. Real-world effectiveness and tolerability of erenumab: A retrospective cohort study. Cephalalgia 2020 Nov;40(13):1511-22.

32. Hoon JD, Hecken AV, Yan L, et al. Phase 1, randomized, double-blind, placebo-controlled, single-dose and multiple dose studies of AMG334 in healthy subjects and migraine patients. Cephalalgia 2015; $35: 45-46$. 